\title{
Can heart rate variation rule out sleep- disordered breathing in heart failure?
}

\author{
A. Vazir*,\#, M. Dayer*,\#, P.C. Hastings*, H.F. Mclntyre" ${ }^{\#}$ M.Y. Henein\#, \\ P.A. Poole-Wilson\# ${ }^{\#}$ M.R. Cowie ${ }^{\#}$, M.J. Morrell* and A.K. Simonds*
}

ABSTRACT: In patients with obstructive sleep apnoea (OSA), the very low frequency power spectral density index (VLFI) derived from analysis of heart rate correlates with the severity of obstructive apnoeas. VLFI is also associated with Cheyne-Stokes respiration/central sleep apnoea (CSR/CSA) in congestive heart failure (CHF). The present authors have tested the hypothesis that per cent VLFI, derived from a standard Holter ECG recording, can be used to detect the presence of OSA and CSR/CSA in patients with mild-to-moderate CHF.

In total, 60 CHF patients underwent polysomnography with monitoring of heart rate. Data from 33 patients were analysed for per cent VLFI. Of the 60 patients, 27 were excluded due to atrial fibrillation, extensive pacing or frequent ventricular extra systoles.

Receiver operator characteristic curves were constructed to establish the per cent VLFI that would optimally identify the presence or absence of sleep-disordered breathing. Using an apnoea-hypopnoea index $>20$ events $\cdot \mathrm{h}^{-1}$ and setting the per cent VLFI at $2.23 \%$ yielded a sensitivity of $85 \%$, specificity of $65 \%$, positive predictive value of $61 \%$ and a negative predictive value of $87 \%$. The latter increased to $100 \%$ when using an apnoea-hypopnoea cut-off of 30 events $\cdot h^{-1}$.

In conclusion, these results suggest that spectral analysis of heart rate may be useful as a "ruleout test" for sleep-disordered breathing in patients with mild-to-moderate congestive heart failure.

KEYWORDS: Central sleep apnoea, congestive heart failure, heart rate variation, obstructive sleep apnoea

$\mathrm{s}$ leep-disordered breathing (SDB) occurs in up to one-half of male patients with congestive heart failure (CHF) [1] and is associated with an accelerated decline in cardiac function and increased morbidity and mortality [2]. Several studies suggest that treatment of SDB is an important adjunct to pharmacotherapy in the management of CHF [3-5]. The diagnosis of SDB may be missed because of a lack of a simple, noninvasive screening test to detect SDB in CHF patients attending cardiology outpatient clinics. In addition, workload pressures on sleep study facilities in many countries make it impractical for all CHF patients to be screened for SDB [6]. This is an important issue as CHF is a common condition, affecting $1-2 \%$ of the general population in developed countries [7].

SDB in CHF comprises of obstructive sleep apnoea (OSA), central sleep apnoea (CSA) and Cheyne-Stokes respiration/periodic breathing (CSR). For simplicity both CSR and CSA will be termed together as CSA/CSR. All three patterns of respiratory disturbance may co-exist in any one patient [8]. Any screening method must be able to pick up all types of SDB.

SDB affects autonomic indices and these alterations can be detected by analysing corresponding variations in heart rate (HR). The variations in $\mathrm{HR}$ (i.e. variations in $\mathrm{R}-\mathrm{R}$ interval) can be detected through power spectral analysis of $R-R$ intervals using Fourier analysis. The latter method has been validated in physiological and pathological conditions [9].

Using Fourier analysis the spectral power changes mainly vary in the: very low (VLF; 0.01-0.05 beat $^{-1}$ ), low (LF: $0.05-0.15$ beat $^{-1}$ ) and high frequency (HF; $0.15-0.40$ beat $^{-1}$ ) bands of the $\mathrm{R}-\mathrm{R}$ intervals. LF band power reflects sympathetic cardiac activation, whereas HF band power reflects parasympathetic vagal cardiac activation [10]. The origins of the VLF are not well defined. However, previous studies have suggested that VLF oscillations are associated with the presence of periodic breathing/CSR in patients with $\mathrm{CHF}$ $[11,12]$. In a more recent study, an increase in
AFFILIATIONS

${ }^{*}$ Clinical and Academic Unit of Sleep and Breathing, and

\#Dept of Cardiology, National Heart and Lung Institute, Imperial College, London, UK

CORRESPONDENCE

A. Simonds

Dept of Sleep and Ventilation

Royal Brompton and Harefield NHS

Trust

Fulham Road

London

SW3 6NP

UK

Fax: 442073518911

E-mail:

a.simonds@rbh.nthames.nhs.uk

Received:

November 152004

Accepted after revision:

October 172005

SUPPORT STATEMENT

A. Vazir and M. Dayer were funded by a British Heart Foundation project grant. M. Morrell was funded by the Wellcome Trust. 
VLF has been shown in patients with OSA compared with controls [13]. The VLF power spectral density of the interbeat interval increment, when corrected for the total power spectral density (per cent VLFI), was strongly associated with the presence of obstructive apnoeas.

The ability of heart rate variability (HRV) analysis to detect SDB in CHF has not been previously assessed. The present authors have tested the hypothesis that per cent VLFI, derived from a standard Holter $24 \mathrm{~h}$ ECG monitor, can be used to detect the presence of clinically important OSA and CSA in patients with varying severity of $\mathrm{CHF}$.

\section{MATERIALS AND METHODS \\ Subjects}

In total, 60 male patients attending cardiology clinics with a diagnosis of $\mathrm{CHF}$ due to idiopathic dilated cardiomyopathy or ischaemic cardiomyopathy were recruited from a wider based study investigating the prevalence of SDB in patients with varying levels of $\mathrm{CHF}$. Selection was not determined by the presence or absence of daytime sleepiness. The selection criteria included recruiting stable CHF patients (defined as no changes in medication for 4 weeks prior to sleep study and no recent hospitalisation), with left ventricular systolic dysfunction (left ventricular ejection fraction $<50 \%$ ) on optimal medical therapy. The patients had to have good lung function (forced expiratory volume in one second/forced vital capacity ratio $>0.70$ ) and no history of chronic neurological disease. The study was approved by the Royal Brompton and Harefield Trust ethics committee (London, UK) and all patients gave written informed consent.

\section{Assessment of heart failure}

All patients underwent a standardised assessment of the functional severity of their heart failure using the New York Heart Association (NYHA) classification and Minnesota living with heart failure score. Patients also underwent transthoracic echocardiography to assess chamber size and left ventricular ejection fraction. The latter was assessed using left ventricular end diastolic and systolic diameters derived from M-Mode echocardiography of the left parasternal long axis view at the level of the tips of the mitral valve. Patients underwent ergometric cardiopulmonary exercise tests (Oxycon; Jaeger Ltd, Hoechberg, Germany) to obtain maximum oxygen uptake $\left(V^{\prime} \mathrm{O}_{2}, \max \right)$ and slope of ventilation in relation to carbon dioxide production (minute ventilation $/ \mathrm{CO}_{2}$ production slope).

\section{Sleep studies}

Full polysomnography was performed using a computerised data acquisition system (Jaeger Sleeplab 1000p; Jaeger Toennies, Wurzburg, Germany). Sleep was monitored using three electroencephalograms (EEG; C4-A1, C3-A2 and O1-A2), two electro-oculograms and a submental electromyogram. Airflow was assessed using a pneumotachograph attached to a facemask (Cranlea \& Co., Birmingham, UK). Arterial oxygen saturation was measured with a finger pulse oximeter (200-E; Nellcor, Pleasanton, CA, USA). Chest and abdominal excursions were monitored using effort bands.

\section{Analysis}

The sleep stage was manually scored using standard criteria [14]. Definitions for respiratory events were as follows: 1)
Obstructive apnoea: complete cessation of air flow with continued paradoxical chest and abdominal movement for $>10$ s. 2) Central apnoea: complete cessation of airflow and complete cessation of chest and abdominal movements for $>10$ s. 3) Hypopnoea: a reduction of airflow of $>50 \%$ from baseline for $>10 \mathrm{~s}$ in association with a $4 \%$ desaturation or EEG arousal. SDB was considered using five cut-off points as an apnoea-hypopnoea index (AHI) of $\geqslant 5,10,15,20$ and 30 events $\cdot h^{-1}$ of sleep. SDB was further classified into either CSA or OSA if $>50 \%$ of the apnoeas were central or obstructive in origin, respectively. The aetiology of hypopnoea could not be determined, therefore, they were categorised as mixed in origin.

\section{Overnight collection of HR data}

HR data were collected with a lightweight portable Holter monitor (Vista; Novacor, Rueil-malmaison, France) sampling at $200 \mathrm{~Hz}$, in concordance with the European Society of Cardiology recommendations on HRV recording [9]. The Holter monitor was manually synchronised with the polysomnography using common time signals. After an overnight ECG recording the Holter monitor was then processed for HRV using a commercial program (Holtersoft; Novacor). This system automates the calculation of per cent VLFI and provides a user-friendly interface for the processing of Holter data. The traces were analysed by one investigator (M. Dayer) blinded to the results of the sleep studies. The period between 00:00-06:00 $\mathrm{h}$ was used for analysis of the HR data. The time spent analysing the trace was limited to $20 \mathrm{~min}$ to ensure that the system would be practical for clinical use. The majority of the $20 \mathrm{~min}$ was used to manually screen the traces for ventricular ectopics, ventricular paced beats and artefacts, which were deleted, so that only valid $\mathrm{R}-\mathrm{R}$ intervals were analysed. This method of editing is recommended by the European Society of Cardiology [9] and has been used in previous studies [15]. After editing the ECG recording, and removing artefact and ectopic beats, the $R-R$ series were then built by concatenation of consecutive sections of valid $R-R$ intervals. The commercial software used for HRV analysis used Fourier transform to analyse the valid R-R series through consecutive 16 min blocks. This gave rise to the VLF power spectral density that is corrected further for total power spectral density to give the per cent VLFI. The calculation of the per cent VLFI takes several seconds. The methodology of identification of the VLF oscillation and per cent VLFI has been extensively developed and described in a previous study by RocHE et al. [13].

For analysis of HRV it was necessary to exclude patients who had atrial fibrillation $(n=18)$. In addition ECG traces in which there were extensive periods of ventricular pacing $(n=2)$, frequent atrial/ventricular ectopics $>10 \%$ of the total number of beats $(n=5)$, or excessive artefact $(n=2)$ were also excluded from HRV analysis. Therefore, 27 patients from the total 60 were excluded. The data on the remaining 33 patients are presented for HRV. The clinical characteristics of these patients are shown in table 1 . The majority of the $33 \mathrm{CHF}$ patients who underwent HRV analysis did not report excessive daytime sleepiness (mean Epworth sleepiness score (ESS) of 8.3; $\mathrm{SD}=4.2)$. 


\begin{tabular}{|c|c|c|}
\hline TABLE 1 & \multicolumn{2}{|c|}{$\begin{array}{l}\text { Clinical characteristics of the males with } \\
\text { congestive heart failure who underwent analysis } \\
\text { of heart rate variation }\end{array}$} \\
\hline \multicolumn{3}{|c|}{ Clinical characteristics } \\
\hline \multicolumn{2}{|l|}{ Males $\mathbf{n}$} & 33 \\
\hline \multicolumn{2}{|c|}{ Ischaemic cardiomyopathy } & $14(42)$ \\
\hline \multicolumn{2}{|l|}{ Diabetes } & $6(18)$ \\
\hline \multicolumn{2}{|l|}{ Age yrs } & $62.1 \pm 11.7$ \\
\hline \multicolumn{2}{|l|}{ Height $\mathbf{c m}$} & $175.1 \pm 8.8$ \\
\hline \multicolumn{2}{|l|}{ Weight kg } & $88.8 \pm 21.2$ \\
\hline \multicolumn{2}{|l|}{$B M I \mathbf{k g} \cdot \mathrm{m}^{-2}$} & $28.8 \pm 5.1$ \\
\hline \multicolumn{2}{|c|}{ Ejection fraction \% } & $37.8 \pm 12.3$ \\
\hline \multicolumn{2}{|c|}{$V^{\prime} \mathrm{O}_{2}, \max \mathrm{mL} \cdot \mathrm{kg}^{-1} \cdot \mathrm{min}^{-1}$} & $17.6 \pm 3.6$ \\
\hline \multicolumn{2}{|c|}{$V^{\prime} \mathrm{E} / V^{\prime} \mathrm{CO}_{2}$ slope } & $32.1 \pm 10.1$ \\
\hline \multicolumn{2}{|l|}{ NYHA class } & $2.1 \pm 0.4$ \\
\hline \multicolumn{2}{|c|}{ Minnesota living with heart failure score } & $25.0 \pm 22.8$ \\
\hline \multicolumn{2}{|c|}{ Beta blockers } & $24(72)$ \\
\hline \multicolumn{2}{|c|}{ ACE inhibitors/angiotensin II receptor antagonists } & $33(100)$ \\
\hline \multicolumn{2}{|l|}{ Diuretics } & $32(96)$ \\
\hline \multicolumn{2}{|l|}{ Digoxin } & $4(12)$ \\
\hline
\end{tabular}

Data are presented as $n$, mean \pm SD or $n(\%)$. BMI: body mass index; $V^{\prime} \mathrm{O}_{2}$, max: maximum oxygen uptake; $V^{\prime} \mathrm{E}$ : minute ventilation; $V^{\prime} \mathrm{CO}_{2}: \mathrm{CO}_{2}$ production; NYHA: New York Heart Association; ACE: angiotensin-converting enzyme.

\section{Statistical analysis}

Values are expressed as mean \pm SD or as median (range). An unpaired t-test was used for normally distributed data and the Mann-Whitney rank sum test when data were not normally distributed. A p-value of $<0.05$ was considered significant. Pearson correlation coefficient was used to examine the association of per cent VLFI and AHI.

Receiver operator characteristics (ROC) [16] curves were constructed to determine the per cent VLFI cut-off that would discriminate between the presence or absence of SDB using five different AHI thresholds of 5,10,15, 20 and 30 events $\cdot \mathrm{h}^{-1}$. The area under curve $(\mathrm{W})$ of the ROC plot evaluated the ability of per cent VLFI to discriminate between the absence and presence of SDB. The minimal $\mathrm{W}$-value 0.5 indicates no discrimination and the maximum $\mathrm{W}$-value of 1.0 indicates perfect discrimination. To assess the accuracy in detecting or excluding the presence of SDB the positive predictive value $(\mathrm{PPV})$, negative predictive value (NPV), and sensitivity and specificity of per cent VLFI were calculated.

\section{RESULTS}

The current authors found a weak but significant $(r=0.52$; $\mathrm{p}=0.002)$ positive correlation between $\mathrm{AHI}$ and the per cent VLFI (fig. 1).

The ROC curves for four of the five different AHI thresholds $\left(10,15,20\right.$ and 30 events $\left.\cdot h^{-1}\right)$ are shown in figure 2 . The ROC curve analysis for the five different AHI thresholds shows that the greatest area under the curve values were obtained when the AHI thresholds of $>30$ and 20 events $\cdot h^{-1}$ were used to define SDB, achieving $\mathrm{W}$-values of 0.88 and 0.82 , respectively, compared with AHI thresholds of 5,10 and 15 events $\cdot h^{-1}$, achieving $W$-values of $0.47,0.57$ and 0.72 , respectively.

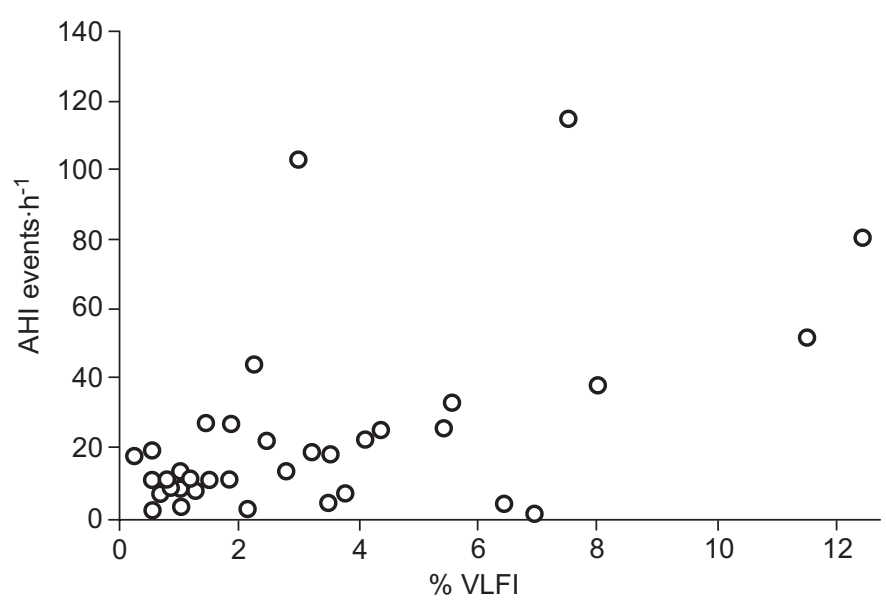

FIGURE 1. Pearson correlation coefficient of apnoea-hypopnoea index (AHI) and very low frequency index (\% VLFI). $R=0.52 ; p=0.002$.

For the AHI threshold of $>20$ events $\cdot \mathrm{h}^{-1}$ setting the per cent VLFI at $2.23 \%$ yielded a sensitivity of $85 \%$ and a specificity of $65 \%$. The PPV was $61 \%$ and the NPV $87 \%$. The NPV and sensitivity increased to $100 \%$ with an AHI threshold of $>30$ events $\cdot h^{-1}$, however, the PPV and specificity values decreased to 37 and $43 \%$, respectively. Therefore, AHI thresholds of $>20$ and 30 events $\cdot h^{-1}$ seem to be valuable thresholds by which per cent VLFI can be used as a test to exclude the presence of significant SDB.

At an AHI threshold $>20$ events $\cdot h^{-1}$, the diagnosis of SDB was established in 13 of the $33 \mathrm{CHF}$ patients. Of these, five had OSA and eight had CSA. Comparisons were made between patients with SDB and patients without SDB and are summarised in table 2. Patients with SDB had a significantly higher per cent VLFI value (4.38 versus $1.49 ; \mathrm{p}=0.003$ ) compared with patients without SDB. However, there was no significant difference with regards to degree of symptoms of $\mathrm{CHF}$ as assessed by the NYHA class, Minnesota living with heart failure scores, and echocardiographical ejection fraction between patients with $\mathrm{SDB}$ versus those without SDB. Interestingly, despite there being no difference in actual $\mathrm{MVO}_{2}$ between $\mathrm{CHF}$ patients with SDB and those without, patients without SDB had a significantly lower per cent predicted $\mathrm{MVO}_{2}$ compared with patients with SDB, suggesting that patients without SDB had a significantly greater degree of exercise limitation. Subjective sleepiness as assessed by the ESS, was not significantly higher in those with SDB, but with a large variation in both groups, suggesting that symptoms of excessive daytime sleepiness alone may not be useful in identifying patients with SDB in this group.

\section{DISCUSSION}

The main findings of the present study were that using a per cent VLFI cut-off of $2.23 \%$ to discriminate between the presence or absence of SDB produced a NPV of $87 \%$ when SDB was defined by an AHI of $>20$ events $\cdot h^{-1}$, and a NPV of $100 \%$ when SDB was defined by an AHI of $>30$ events $\cdot h^{-1}$. These results suggest that spectral analysis of HR may be used as a rule-out test to exclude the presence of serious SDB in patients with mild-to-moderate congestive heart failure. 

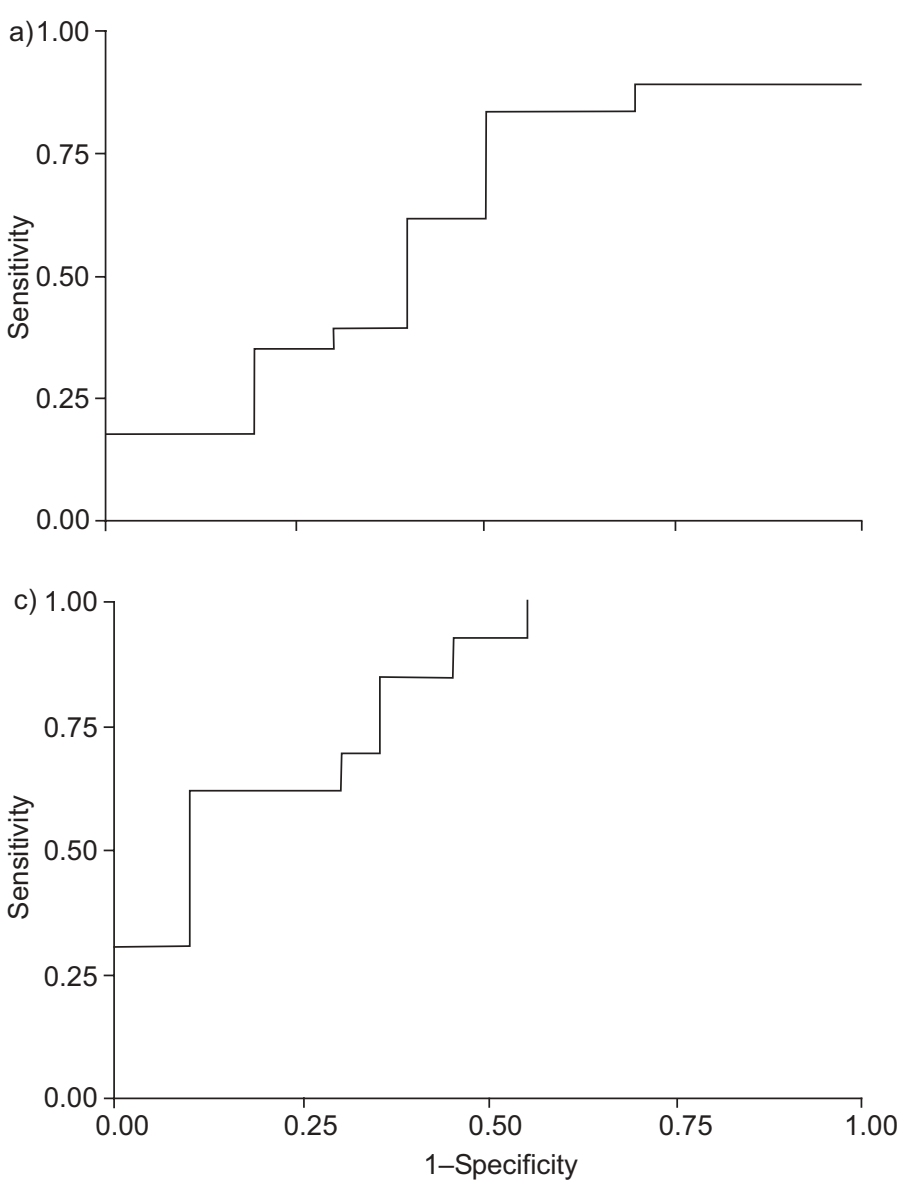

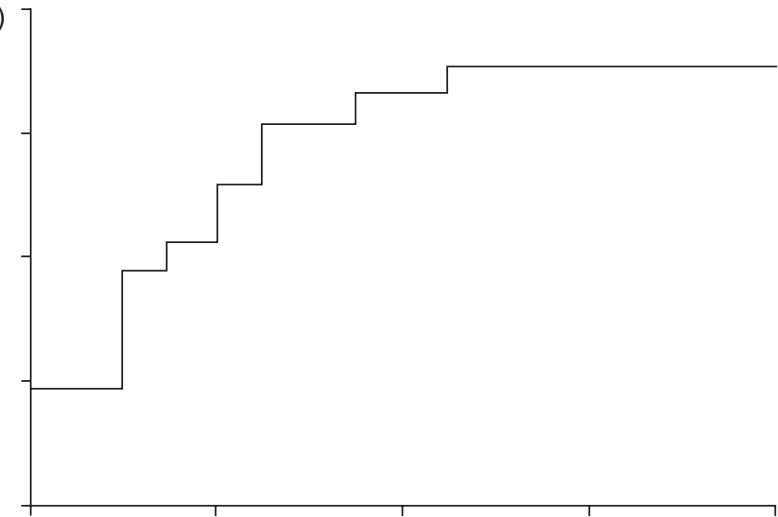

d)

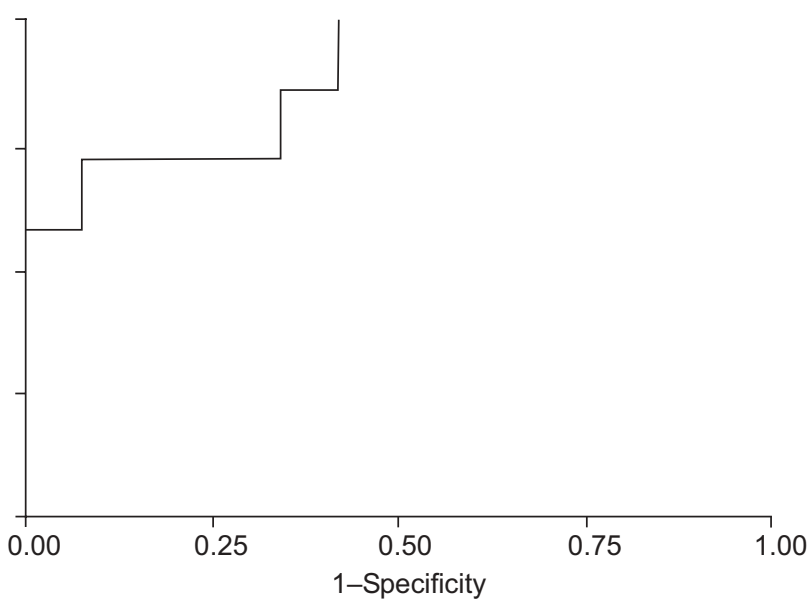

FIGURE 2. Receiver operator characteristic curves for assessing the ability of very low frequency index to discriminate between the presence or absence of sleepdisordered breathing using four different apnoea-hypopnoea index thresholds. a) 10, b) 15, c) 20 and d) 30 events $\cdot h^{-1}$. The areas under the curve were $0.61,0.72,0.82$ and 0.88 , respectively. The p-values designated the asymptotic significance and were $0.390,0.030,0.030$ and 0.002 , respectively. The standard error was $0.110,0.100,0.073$ and 0.073 , respectively.

In the current study, per cent VLFI was used to identify CHF patients with OSA and CSA/CSR. The majority of patients within the present study, who had SDB, mainly had central apnoeas plus hypopnoea, which may have been more difficult to detect. However, despite these potential issues, the current findings are similar to those of RocHE et al. [17], who found that per cent VLFI was particularly strongly associated with the presence of obstructive apnoeas within the general population and could, therefore, identify the presence or absence of OSA.

The increase in VLF in OSA may result from respiratory effort against upper airway occlusion, associated first with a strong increase in parasympathetic autonomic nervous activity followed by an abrupt sympathetic activation due to the consequent hypoxia [18], a sequence which dramatically enhances $\mathrm{R}-\mathrm{R}$ variability. These responses may be a reflection of the interaction between the abnormal cyclical responses of chemo- and baroreceptor reflexes. The latter responses are the thought to be the cause for the oscillations at VLF seen in respiration during CSA/CSR and periodic breathing. These VLF oscillations seen in the respiration of patients with CSA/ CSR correspond to VLF oscillations seen in HR and blood pressure (BP) [19]. A study by LEUNG et al. [20] demonstrated that power spectral analysis revealed prominent oscillation in $\mathrm{R}-\mathrm{R}$ interval and BP precisely at the same frequency as CSR, such that HR and BP peaked during the ventilatory phase and reached a nadir during apnoea. Inhalation of $\mathrm{CO}_{2}$ abolished CSR and caused regular breathing by raising the arterial $\mathrm{CO}_{2}$, this in turn abolished the oscillations seen with HR and BP, suggesting that changes in tidal volume are responsible for the variation in $\mathrm{R}-\mathrm{R}$ interval. This has been supported by studies in healthy awake volunteers [21].

In the present study, the majority of the patients had mild-tomoderate symptomatic CHF. This group constituted of $70 \%$ of $\mathrm{CHF}$ patients from within the community [22]. However, a significant proportion of patients with severe $\mathrm{CHF}$ due to severe left ventrical systolic dysfunction have reduced HRV $[23,24]$, which theoretically could generate abnormally high values of per cent VLFI. This could produce false positive results when screening for SDB with HRV analysis. A recent study by HADASE et al. [25] demonstrated that the VLF component of HRV is a powerful predictor of cardiac-related mortality and rehospitalisation in patients with mild-to-severe $\mathrm{CHF}$. However, the occurrence of SDB was not noted in that study and it is possible that the increased VLF could have occurred due to the presence of SDB.

Previous studies have shown that time domain variables may also be useful in identifying OSA [13, 26]. However, the 


\begin{tabular}{|c|c|c|c|c|}
\hline \multirow[t]{2}{*}{ TABLE 2} & \multicolumn{4}{|c|}{$\begin{array}{l}\text { Characteristics of congestive heart failure } \\
\text { patients with and without sleep-disordered } \\
\text { breathing (SDB) }\end{array}$} \\
\hline & & Non-SDB' & SDB $^{+}$ & $p$-value \\
\hline \multicolumn{2}{|c|}{ AHI events $\cdot h^{-1}$} & $9.2(0-19.1)$ & $32.6(21.8-114)$ & $<0.001$ \\
\hline \multicolumn{2}{|c|}{ VLFI \% } & $1.49(0.3-6.9)$ & $4.38(1.45-12.4)$ & 0.003 \\
\hline \multicolumn{2}{|l|}{ NYHA } & $2(2-3)$ & $2(2-3)$ & 0.810 \\
\hline \multicolumn{2}{|c|}{ Minnesota score } & $26.9 \pm 21.0$ & $34.8 \pm 24$ & 0.326 \\
\hline \multicolumn{2}{|c|}{ Ejection fraction \% } & $40.2 \pm 12.3$ & $34.9 \pm 11.6$ & 0.227 \\
\hline \multicolumn{2}{|c|}{$\begin{array}{l}\text { Actual } V^{\prime} \mathrm{O}_{2}, \max \\
\mathrm{mL} \cdot \mathrm{kg}^{-1} \cdot \mathrm{min}^{-1}\end{array}$} & $16.5 \pm 3.4$ & $17.8 \pm 4.7$ & 0.387 \\
\hline \multicolumn{2}{|c|}{$V^{\prime} \mathrm{O}_{2}$, max \% predicted } & $63.5 \pm 12.0$ & $76.1 \pm 15.98$ & 0.020 \\
\hline \multicolumn{2}{|c|}{$V^{\prime} \mathrm{E} / V^{\prime} \mathrm{CO}_{2}$ slope } & $29.8(22.4-69.1)$ & $27.3(25.1-38.7)$ & 0.637 \\
\hline \multicolumn{2}{|l|}{ Age yrs } & $61.4 \pm 11.9$ & $62.4 \pm 9.4$ & 0.798 \\
\hline \multicolumn{2}{|l|}{$\mathrm{BMI} \mathbf{k g} \cdot \mathrm{m}^{-2}$} & $28.5(21.5-39.5)$ & $31.1(24.7-45.0)$ & 0.045 \\
\hline \multicolumn{2}{|c|}{ Epworth sleepiness score } & $6.5(3-16)$ & $9.0(2-18)$ & 0.231 \\
\hline
\end{tabular}

Data are presented as mean \pm SD or median (range), unless otherwise stated. AHI: apnoea-hypopnoea index; VLFI: very low frequency index; NYHA: New York Heart Association; $V^{\prime} \mathrm{O}_{2}$, max: maximum oxygen uptake; $V^{\prime} E$ : minute

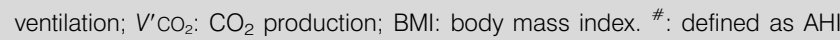
$>20$ events $\cdot h^{-1} ; \bullet: n=20 ;{ }^{+}: n=13 ;{ }^{\text {s: }} p<0.05$ was deemed statistically significant

advantage of per cent VLFI (a frequency domain variable), is that analysis is easier, quicker and not dependent on obtaining and separating day and night time ECG recordings, as is required when using the time domain variables. Thus, for obtaining a per cent VLFI value, an overnight ECG recording is sufficient as in the current study.

The presence of periodic limb movements (PLM) during sleep are known to affect HR [27-29], however the effect of PLM on VLF is unknown. In the present study, of the 33 patients who underwent HRV analysis 15 had PLM (defined by PLM index $>5$ events $\cdot \mathrm{h}^{-1}$ ) and of the 13 patients with SDB (defined by AHI $>20$ events $\cdot h^{-1}$ ), four had coexisting PLM. Further research is required to determine the effect of PLM on per cent VLFI.

The key limitation to the use of HRV as a rule out test for SDB in patients with CHF is that it cannot be used in patients who are paced extensively throughout the night, and those with frequent ectopy or atrial fibrillation (AF). In the current study, $42 \%$ ( 25 out of 60 ) of patients were excluded for these reasons, 18 had AF, five had frequent ventricular ectopy and two were extensively paced. Of the 27 patients, 10 had significant SDB with AHI $>20$ events $\cdot h^{-1}$ (CSA: eight patients; OSA: two patients), with eight cases of SDB occurring in the $18 \mathrm{AF}$ patients. It can be argued that CHF patients with AF should be considered for a sleep study without pre-selection by screening, as AF is a powerful risk factor for SDB, in particular CSA [30]. The presence of AF is also common in individuals with OSA [31], where it has been demonstrated that persistent obstructive apnoeas can increase the recurrence of AF after successful cardioversion. However, $10-30 \%$ of $\mathrm{CHF}$ patients may have AF [32-34], thus, sleep laboratories may become overburdened and alternative methods of screening for SDB, such as overnight pulse oximetry [35], may be required for
CHF patients in AF and for those CHF patients in which HRV analysis is not possible. SERIES et al. [36] have recently shown that pulse oximetry in heart failure is a very useful method of identifying SDB. They demonstrated that home pulse oximetry, using desaturation of $>2 \%$, had a sensitivity of $85 \%$ and specificity of $93 \%$ for detecting SDB in patients with $\mathrm{CHF}$ irrespective of underlying cardiac rhythm. In their series, home pulse oximetry missed the diagnosis of SDB in five out of 33 patients with SDB when compared with polysomnography. The latter may be related to the fact that conventional pulse oximetry may not always detect patients with CSA as they may not desaturate, and also pulse oximetry may underestimate the severity of the SDB. Spectral analysis of pulse oximetry [37] may be more accurate at detecting SDB then conventional measurements of pulse oximetry, such as desaturation index or time spent below an arterial oxygen saturation measured by pulse oximetry of $90 \%$. Furthermore, the addition of nocturnal HRV to pulse oximetry, so that there is simultaneous ECG and oximetry monitoring, could provide a more powerful screening tool to detect both CSA and OSA, as this will take into account patients who are excluded from HRV analysis, as in the current study group of patients.

The main advantage of HRV is that it can not only be used as a potential screen for SDB, but is readily available to cardiologists as $24 \mathrm{~h}$ Holter monitoring is routinely performed, given the high incidence of arrhythmias in CHF. Furthermore, cardiologists are in a position to analyse the $24 \mathrm{~h}$ ECG recording for per cent VLFI, thereby concurrently screening for SDB. Thus, HRV analysis is probably most useful in the cardiology setting.

The present authors have shown that per cent VLFI can be useful in identifying patients who should be investigated further for SDB, and excluding individuals in which a sleep study is not necessary. CHF patients, in whom per cent VLFI suggests the presence of SDB, should undergo a sleep study to determine the type of SDB, as per cent VLFI is unable to distinguish between CSA and OSA. Determining the type of SDB is important, as the treatment of CSA and OSA does differ. The treatment of the latter in $\mathrm{CHF}$ patients is well established with continuous positive airway pressure therapy $[4,5]$. However, the optimal treatment of CSA remains unclear at the present time.

The current authors have proposed a potential "intend to diagnose" protocol that is outlined in figure 3 . In the group of patients who are eligible for HRV analysis, if they have a per cent VLFI of $>2.23$ they are at risk of SDB and should go on to have a sleep study. Those with a per cent VLFI value of $<2.23$ are less likely to have SDB, but in the presence of symptoms of $\mathrm{SDB}$ (e.g. excessive daytime sleepiness) or risk factors, such as obesity, large neck circumference and retrognathia, should go on to have a sleep study. As already argued, individuals with $\mathrm{AF}$ are at an increased risk of SDB, especially CSA, and also individuals with excessive ventricular ectopy or extensive pacing in the presence of symptoms or risk factors of SDB should be considered for a sleep study or an alternative screening method should be sought as discussed above.

In using a per cent VLFI threshold of 2.23, and defining SDB as an AHI $>30$ events $\cdot h^{-1}$, patients with severe SDB would not 


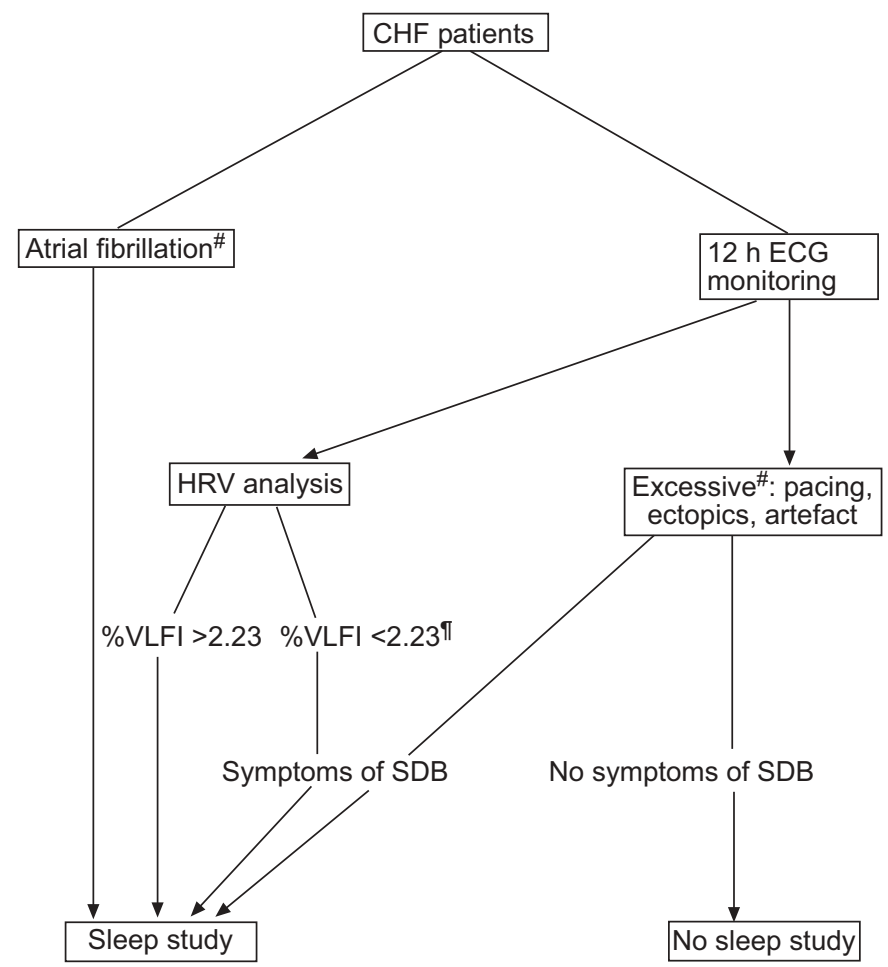

FIGURE 3. Schematic diagram to show an "intend to diagnose" protocol for the use of heart rate variability (HRV) in ruling out sleep-disordered breathing (SDB) in patients with congestive heart failure (CHF). VLFI: very low frequency index. \#: patients in whom HRV analysis is not useful (atrial fibrillation, excessive ventricular pacing, ectopics or artefact) may be screened using an alternative, such as pulse oximetry; ": patients with per cent VLFI $<2.23$ only progress to sleep study if they have symptoms of SDB, i.e. witnessed apnoeas or excessive snoring.

have been missed. However, using the same per cent VLFI threshold, but defining SDB as an AHI $>20$ events $\cdot h^{-1}$, the presence of SDB would have been missed in two out of the 33 patients who underwent HRV analysis. Lowering the AHI threshold further to 15 events $\cdot h^{-1}$, but continuing to use a per cent VLFI of 2.23, the presence of SDB would have been missed in a total of four patients. However, all four patients had relatively elevated ESS (12, 11, 11 and 9, respectively). Therefore, by combining HRV analysis with additional screening for symptoms or risk factors of SDB, all four patients would have undergone a sleep study. With the use of per cent VLFI as a rule-out test, in the protocol shown in figure 3, the current authors would have saved 16 sleep studies (just under 26\%) from the 60 patients, taking account those individuals with a per cent VLFI $<2.23$ who would have had sleep studies because of the presence of symptoms or risk factors of SDB.

In conclusion, in the majority of congestive heart failure patients with mild-to-moderate symptoms, the analysis of nocturnal heart rate variability by spectral analysis is a quick, easy and a promising screening tool for sleep-disordered breathing. Setting the per cent very low frequency index at $2.23 \%$ provided a high negative predictive value that was necessary for a rule-out test for sleep-disordered breathing in the present study, both for central and obstructive sleep apnoea. However, as with any clinical test, these results require prospective validation using a separate sample. For health services, particularly cardiac services, the use of heart rate variability analysis from routine Holter monitoring over a 24-h or overnight period may reduce demands on overburdened sleep laboratory facilities by identifying patients with heart failure who are unlikely to have sleep-disordered breathing.

\section{ACKNOWLEDGEMENTS}

The authors would like to thank Novacor (Rueil-malmaison, France) for providing the heart rate monitor and analysis software.

\section{REFERENCES}

1 Javaheri S, Parker TJ, Liming JD, et al. Sleep apnea in 81 ambulatory male patients with stable heart failure. Types and their prevalences, consequences, and presentations. Circulation 1998; 97: 2154-2159.

2 Hanly PJ, Zuberi-Khokhar NS. Increased mortality associated with Cheyne-Stokes respiration in patients with congestive heart failure. Am J Respir Crit Care Med 1996; 153: 272-276.

3 Sin DD, Logan AG, Fitzgerald FS, Liu PP, Bradley TD. Effects of continuous positive airway pressure on cardiovascular outcomes in heart failure patients with and without Cheyne-Stokes respiration. Circulation 2000; 102: 61-66.

4 Kaneko Y, Floras JS, Usui K, et al. Cardiovascular effects of continuous positive airway pressure in patients with heart failure and obstructive sleep apnea. N Engl J Med 2003; 348: 1233-1241.

5 Mansfield DR, Gollogly NC, Kaye DM, Richardson M, Bergin P, Naughton MT. Controlled trial of continuous positive airway pressure in obstructive sleep apnea and heart failure. Am J Respir Crit Care Med 2004; 169: 361-366.

6 Flemons WW, Douglas NJ, Kuna ST, Rodenstein DO, Wheatley J. Access to diagnosis and treatment of patients with suspected sleep apnea. Am J Respir Crit Care Med 2004; 169: 668-672.

7 Cowie MR, Mosterd A, Wood DA, et al. The epidemiology of heart failure. Eur Heart J 1997; 18: 208-225.

8 Tkacova R, Niroumand M, Lorenzi-Filho G, Bradley TD. Overnight shift from obstructive to central apneas in patients with heart failure: role of $\mathrm{PCO}_{2}$ and circulatory delay. Circulation 2001; 103: 238-243.

9 Heart rate variability. Standards of measurement, physiological interpretation, and clinical use. Task Force of the European Society of Cardiology and the North American Society of Pacing and Electrophysiology. Eur Heart J 1996; 17: 354-381.

10 Malik M, Farrell T, Cripps T, Camm AJ. Heart rate variability in relation to prognosis after myocardial infarction: selection of optimal processing techniques. Eur Heart J 1989; 10: 1060-1074.

11 Ichimaru Y, Yanaga T. Frequency characteristics of the heart rate variability produced by Cheyne-Stokes respiration during 24-hr ambulatory electrocardiographic monitoring. Comput Biomed Res 1989; 22: 225-233.

12 Mortara A, Sleight P, Pinna GD, et al. Abnormal awake respiratory patterns are common in chronic heart failure and may prevent evaluation of autonomic tone by measures of heart rate variability. Circulation 1997; 96: 246-252. 
13 Roche F, Duverney D, Court-Fortune I, et al. Cardiac interbeat interval increment for the identification of obstructive sleep apnea. Pacing Clin Electrophysiol 2002; 25: 1192-1199.

14 Rechtschaffen A, Kales A. A manual of standardised terminology, techniques and scoring systems for sleep stages of human subjects. Publication No. 204. Washington, National Institute of Health, 1968.

15 Boveda S, Galinier M, Pathak A, et al. Prognostic value of heart rate variability in time domain analysis in congestive heart failure. J Interv Card Electrophysiol 2001; 5: 181-187.

16 Zweig MH, Campbell G. Receiver-operating characteristic (ROC) plots: a fundamental evaluation tool in clinical medicine. Clin Chem 1993; 39: 561-577.

17 Roche F, Sforza E, Duverney D, et al. Heart rate increment: an electrocardiological approach for the early detection of obstructive sleep apnoea/hypopnoea syndrome. Clin Sci (Lond) 2004; 107: 105-110.

18 Guilleminault C, Connolly S, Winkle R, Melvin K, Tilkian A. Cyclical variation of the heart rate in sleep apnoea syndrome. Mechanisms, and usefulness of $24 \mathrm{~h}$ electrocardiography as a screening technique. Lancet 1984; 1: 126-131.

19 Ichimaru Y, Yanaga T. Frequency characteristics of the heart rate variability produced by Cheyne-Stokes respiration during 24-hr ambulatory electrocardiographic monitoring. Comput Biomed Res 1989; 22: 225-233.

20 Leung RS, Floras JS, Lorenzi-Filho G, Rankin F, Picton P, Bradley TD. Influence of Cheyne-Stokes respiration on cardiovascular oscillations in heart failure. Am J Respir Crit Care Med 2003; 167: 1534-1539.

21 Lorenzi-Filho G, Dajani HR, Leung RS, Floras JS, Bradley TD. Entrainment of blood pressure and heart rate oscillations by periodic breathing. Am J Respir Crit Care Med 1999; 159: 1147-1154.

22 Davies M, Hobbs F, Davis R, et al. Prevalence of leftventricular systolic dysfunction and heart failure in the Echocardiographic Heart of England Screening study: a population based study. Lancet 2001; 358: 439-444.

23 Ponikowski P, Anker SD, Chua TP, et al. Depressed heart rate variability as an independent predictor of death in chronic congestive heart failure secondary to ischemic or idiopathic dilated cardiomyopathy. Am J Cardiol 1997; 79: 1645-1650.

24 La Rovere MT, Pinna GD, Maestri R, et al. Short-term heart rate variability strongly predicts sudden cardiac death in chronic heart failure patients. Circulation 2003; 107: 565-570.

25 Hadase M, Azuma A, Zen K, et al. Very low frequency power of heart rate variability is a powerful predictor of clinical prognosis in patients with congestive heart failure Circ J 2004; 68: 343-347.

26 Roche F, Gaspoz JM, Court-Fortune I, et al. Screening of obstructive sleep apnea syndrome by heart rate variability analysis. Circulation 1999; 100: 1411-1415.

27 Sforza E, Juony C, Ibanez V. Time-dependent variation in cerebral and autonomic activity during periodic leg movements in sleep: implications for arousal mechanisms. Clin Neurophysiol 2002; 113: 883-891.

28 Sforza E, Jouny C, Ibanez V. Time course of arousal response during periodic leg movements in patients with periodic leg movements and restless legs syndrome. Clin Neurophysiol 2003; 114: 1116-1124.

29 Winkelman JW. The evoked heart rate response to periodic leg movements of sleep. Sleep 1999; 22: 575-580.

30 Sin DD, Fitzgerald F, Parker JD, Newton G, Floras JS, Bradley TD. Risk factors for central and obstructive sleep apnea in 450 men and women with congestive heart failure. Am J Respir Crit Care Med 1999; 160: 1101-1106.

31 Kanagala R, Murali NS, Friedman PA, et al. Obstructive sleep apnea and the recurrence of atrial fibrillation. Circulation 2003; 107: 2589-2594.

32 Carson PE, Johnson GR, Dunkman WB, Fletcher RD, Farrell L, Cohn JN. The influence of atrial fibrillation on prognosis in mild to moderate heart failure. The V-HeFT Studies. The V-HeFT VA Cooperative Studies Group. Circulation 1993; 87: Suppl. 6, VI102-VI110.

33 Dries DL, Exner DV, Gersh BJ, Domanski MJ, Waclawiw MA, Stevenson LW. Atrial fibrillation is associated with an increased risk for mortality and heart failure progression in patients with asymptomatic and symptomatic left ventricular systolic dysfunction: a retrospective analysis of the SOLVD trials. Studies of Left Ventricular Dysfunction. J Am Coll Cardiol 1998; 32: 695-703.

34 Stevenson WG, Stevenson LW, Middlekauff HR, et al. Improving survival for patients with atrial fibrillation and advanced heart failure. J Am Coll Cardiol 1996; 28: 1458-1463.

35 Staniforth AD, Kinnear WJ, Starling R, Cowley AJ. Nocturnal desaturation in patients with stable heart failure. Heart 1998; 79: 394-399.

36 Series F, Kimoff RJ, Morrison D, et al. Prospective evaluation of nocturnal oximetry for detection of sleeprelated breathing disturbances in patients with chronic heart failure. Chest 2005; 127: 1507-1514.

37 Zamarron C, Romero PV, Rodriguez JR, Gude F. Oximetry spectral analysis in the diagnosis of obstructive sleep apnoea. Clin Sci (Lond) 1999; 97: 467-473. 